\title{
Vulnerabilidad energética en la metrópoli de Buenos Aires
}

Energy Vulnerability in Buenos Aires Metropolis

Vulnerabilidade energética na metrópole de Buenos Aires

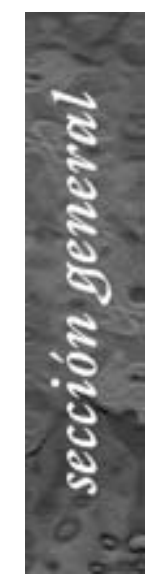

\section{Silvina Carrizo * \\ Marie Noelle Carre ${ }^{* *}$ \\ Jean Igor Michaux ${ }^{\star * *}$}

Recibido: 12 de enero de 2013

Aprobado: 25 de octubre de 2013

Doi: dx.doi.org/10.12804/territ30.2014.06

Para citar este artículo:

Carrizo, S., Carre, M. N. y Michaux, J. I. (2014). Vulnerabilidad energética en la metrópoli de Buenos Aires. Territorios, 30, 127-146. doi: dx.doi.org/10.12804/territ30.2014.06

\begin{abstract}
* Arquitecta. Diplôme d'études approfondies y doctor en Ordenamiento territorial. Investigador adjunto Conicet, Centro de Estudios Urbanos y Regionales, profesor adjunto Unnoba Universidad Nacional del Noroeste de la Provincia de Buenos Aires. Correo electrónico: scarrizo@conicet.gov.ar

** Geógrafa. Master 2 Sistemas territoriales, Desarrollo Sustentable. Docente auxiliar Université Sorbonne Nouvelle Paris 3 IhealCreda. Correo electrónico: marie-noelle.carre@univparis3.fr

** Economista. Bachelor en Relaciones Internacionales y Máster en Economía del Desarrollo. Correo electrónico:jimichaux@aol.com
\end{abstract}


Palabras clave

Vulnerabilidad, energía, región metropolitana.

Keywords

Vulnerability, energy, metropolitan region.

Palavras-chave

Vulnerabilidade, energia, região metropolitana.

\section{territarias 30} 128

\section{RESUMEN}

Argentina declaró el uso racional y la eficacia energética una prioridad nacional, en consonancia con las recomendaciones de la Agencia Internacional de la Energía. Buenos Aires se ha comprometido con varios programas internacionales de preparación al cambio climático. Así a distintas escalas surgen iniciativas para modificar las condiciones y modalidades de aprovisionamiento y consumo energéticos, que revelan la precariedad y vulnerabilidad del sistema y los servicios. Este trabajo presenta una reflexión sobre los cambios que se dan en ese sentido, mostrando los inconvenientes del modelo energético argentino en Buenos Aires y las estrategias o medidas que tienden a revertirlos y a preparar la ciudad para situaciones críticas. En general, se trata de iniciativas que se implementan individual o parcialmente, sin que haya un plan integral promovido a escala colectiva. Las correcciones resultan marginales en el conjunto sin aliviar los problemas que se agravan por el crecimiento vertiginoso de las demandas. Entonces la continuidad del modelo energético argentino basado en hidrocarburos, para abastecer infraestructura que trabaja al límite de su capacidad y servir territorios que no se han preparado para las posibles crisis, explicaría la vulnerabilidad del sistema de abastecimiento metropolitano.

\section{ABSTRACT}

In line with recommendations of the International Energy Agency, Argentina has recently made energy efficiency a national priority. Buenos Aires is committed to various international programs to rationalize the use of energy and tackle growing Climate Change challenges. Initiatives to modify the terms and conditions of energy supply and consumption are appearing at different scales, revealing the precariousness and vulnerability of the whole system. Thus, this paper sheds light on the main drawbacks of Argentine Energy Model and it presents some of the changes taking place regarding these issues. It focuses on strategies and measures promoted to prepare Buenos Aires metropolitan region for critical situations. In general initiatives are implemented individually and they result insufficient to meet the challenges. The continuity of Argentine Energy Model based on the intensive use of fossil fuels that serve infrastructure functioning near its maximum capacity and territories not prepared for potential crises, could explain metropolitan energy system vulnerability.

\section{RESUMO}

A Argentina declarou o uso racional e a eficácia energética uma prioridade nacional, em consonância com as recomendações da Agencia Internacional da Energia. Buenos Aires tem se comprometido com vários programas internacionais de preparação à mudança climática. Assim a diferentes escalas surgem iniciativas para modificar as condições e modalidades de aprovisionamento e consumo energéticos, que revelam a precariedade e vulnerabilidade do sistema e os serviços. Este trabalho apresenta uma reflexão sobre as mudanças que se dão nesse sentido, mostrando os inconvenientes do modelo energético argentino em Buenos Aires e as estratégias ou medidas que tendem a revertêlos e a preparar a cidade para situações críticas. Em geral, trata-se de iniciativas que se implementam individual ou parcialmente, sem que exista um plano integral promovido a escala coletiva. As correções resultam marginais no conjunto sem aliviar os problemas que se agravam pelo crescimento vertiginoso das demandas. Então, a continuidade do modelo energético argentino baseado em 
hidrocarbonatos, para abastecer infraestrutura que trabalha ao limite de sua capacidade e servir territórios que não têm se preparado para as possíveis crises, explicaria a possível vulnerabilidade do sistema de abastecimento metropolitano.

\section{Introducción}

En los últimos diez años, Buenos Aires se ha comprometido con varios programas internacionales de preparación al cambio climático. Entre ellos, el Pacto Climático Global de Ciudades - conocido como Pacto de la Ciudad de México- concebido para la Cumbre Climática Mundial de Alcaldes en la Ciudad de México, en noviembre de 2010 y el C40 Cities Climate Leadership Group, una red de ciudades que, desde 2005, actúa en la reducción de emisiones de gases de efecto invernadero, asociada en 2006, a la Clinton Climate Initiative y, en 2011, al Carbon Disclosure Project que funciona desde 2000, midiendo y divulgando el impacto de empresas y ciudades en el ambiente. Dentro de un amplio panel de iniciativas, la participación de la ciudad al programa "Desarrollando ciudades resilientes. Mi ciudad se está preparando" lanzado por Naciones Unidas en 2010 , apunta a prevenir en la metrópoli, las posibles catástrofes y desastres derivados de cambios atmosféricos de temperatura y humedad. Pero, como diría Hilda Hertzer (1990), "los desastres no son tan naturales como parecen..." sino que los desastres desencadenados por un fenómeno natural, resultan de procesos sociales, económicos y políticos. Por ende Buenos Aires, como otras ciudades que buscan integrar el círculo de las urbes globales, trata de enfrentar las amenazas naturales (tsunamis, terremotos, sequías, inundaciones, incendios, erupciones volcánicas, huracanes o deslizamientos), así como también intenta afrontar una gobernanza metropolitana débil y un desarrollo urbano no controlado (aumento de población y densidad urbana, concentración de activos económicos, escasez de suelo, construcciones inapropiadas, degradación de los ecosistemas y accidentes en transporte o explosiones). Estas acciones humanas constituyen factores amplificadores de la vulnerabilidad, la que entendemos como la condición por la que una sociedad podría sufrir pérdidas o daños, y se define tanto por el entorno natural como por el social, político y económico y queda sujeta a cambios (Blaikie, Cannon, Davis y Wisner, 1996).

En materia de vulnerabilidad, la dimensión territorial resulta clave. Como plantean Robert d'Ercole y Pascale Metzger, geógrafos especialistas en riesgos (2009), "la vulnerabilidad territorial sugiere que en cualquier territorio existen elementos localizables capaces de generarle vulnerabilidad y difundirla, produciendo efectos que pueden perturbar, comprometer o interrumpir su funcionamiento y desarrollo. En esa lógica, el análisis de la vulnerabilidad territorial apunta a identificar, caracterizar y jerarquizar los espacios a partir de los cuales territarias 30

129 
1 Se define como región metropolitana la Ciudad Autónoma de Buenos Aires y un conjunto de 34 municipios de la Provincia de Buenos Aires que la rodean, incluyendo a los 24 partidos del Gran Buenos Aires.

\section{territarias 30} 130 se crea y se difunde la vulnerabilidad en los territorios". Este marco de análisis postula que tomar en cuenta la vulnerabilidad diferenciada de los territorios es un primer paso hacia la prevención de los riesgos. Siguiéndolo, nos parece que para el caso de Buenos Aires, ante los problemas de resiliencia metropolitana frente a las crisis vinculadas con el cambio climático, resulta adecuado y conveniente reflexionar antes sobre la vulnerabilidad energética. Entendemos que la vulnerabilidad de este sistema no equivale a la de la metrópoli, pero resulta clave y se encuentra relativamente poco estudiada.

Las redes energéticas constituyen piezas estratégicas en la definición de vulnerabilidad metropolitana, ya que pueden acarrear condiciones de inseguridad y riesgos asociados para la vida de los habitantes - como motores de sus actividades cotidianas o esenciales - y de la ciudad - como sistema, nexo imprescindible para las actividades articuladas al espacio global- En sí, el sistema energético constituye uno de los elementos más vulnerables a las catástrofes. En Nueva York, el paso del huracán Sandy (2012) lo puso en evidencia. Al neutralizar el funcionamiento de un generador eléctrico en Manhattan, provocó el cierre momentáneo de uno de los centros bursátiles electrónicos más potentes del mundo, lo que impactó la velocidad y la intensidad de los intercambios globales. Frente a escenarios de este tipo, las recomendaciones formuladas por organismos internacionales, como las Naciones Unidas, alientan a las ciudades a adoptar medidas que favorezcan la resiliencia urbana: diversificar sus fuentes de energía, aumentar la flexibilidad del sistema, hacer un uso racional de los recursos y lograr más equidad en el acceso a la energía. Estas medidas se pueden acoplar a las estrategias de mitigación del cambio climático que promueven especialmente la incorporación de energías renovables y la eficiencia energética.

En este contexto, este artículo pretende hacer un aporte a los debates sobre vulnerabilidad metropolitana y adaptación al cambio climático, como problemas de fenómenos naturales y gobernanza, poco tratados para las ciudades latinoamericanas y escasamente vistos desde las problemáticas de la energía, a pesar de que esta sea factor importante de vulnerabilidad y fuerte emisora de dióxido de carbono. Para ello elaboramos un panorama de situación energética actual y de tendencias al cambio (o continuidades) en el espacio metropolitano de Buenos Aires, que encuentra problemas de abastecimiento cada vez más agudos y al que se le plantean grandes desafíos a mediano y largo plazo. Buenos Aires aglutina trece millones de habitantes - más de un tercio de la población argentina (Indec, 2010) - repartidos en varias y diversas entidades administrativas. ${ }^{1}$ Este desafío de fragmentación y superposición política, lo comparte con la mayoría de las grandes urbes del mundo, pero con la especificidad latinoamericana de los problemas de inequidad en la distribución de los recursos y del acceso a los servicios urbanos.

La composición de ese panorama de vulnerabilidad energética y estrategias de cambio implica develar la gran complejidad 
en términos de actores, de niveles de gobierno y de modos de accionar. Este trabajo se funda en un seguimiento regular de la problemática, realizado en el marco de proyectos individuales y colectivos de investigación y focalizado en la metrópoli desde 2010. El proyecto Termos ${ }^{2}$ e investigaciones de maestría y de doctorado en geografía brindaron recursos económicos y abrieron oportunidades de estudio, intercambio y reflexión para desarrollar el análisis. En esos marcos se realizaron trabajos de campo con visitas a instalaciones y entrevistas semiestructuradas a actores del sector de energía (generadores eléctricos; distribuidores de gas y electricidad; espacios de recolección y tratamiento de residuos; funcionarios públicos nacionales, provinciales y de la ciudad; ONG) y a investigadores especializados en problemáticas afines. Se recopiló material periodístico, profesional y académico, con información cuantitativa, cualitativa y espacial. El trabajo tiene, por ende, una fuerte base empírica. La metodología retenida es pluridisciplinaria, aunque se apoya especialmente en la geografía, fundamentándose en las lógicas espaciales con saltos de escalas y profundización de los vínculos entre ellas, así como también en los análisis espaciales y temporales en distintas dimensiones territoriales - sociales, económicas, político institucionales o ambientales(Di Meo, 1998). Siguiendo este tratamiento, el artículo se estructura en dos partes.

La primera parte explica el funcionamiento del sistema energético metropolitano de Buenos Aires en resonancia con los cambios en las redes nacionales a principios del siglo XXI. Muestra la vulnerabilidad energética metropolitana frente a la falta de seguridad en el sistema nacional por la escasez de recursos, la dependencia creciente de abastecimientos externos, las dificultades para la expansión de actividades energéticas (desde la extracción de hidrocarburos hasta la generación eléctrica o la distribución de gas y combustibles) y la utilización ineficiente de los recursos (consumo).

La segunda parte muestra cambios que se introducen, con el fin de contribuir a contener las dificultades y mejorar el funcionamiento. Se presentan entonces iniciativas recientes que demuestran cómo las discusiones internacionales sobre resiliencia urbana y adaptación al cambio climático generaron experiencias sustentables. Se trata particularmente de la promoción de fuentes alternativas y del uso más eficiente de la energía. Sin embargo, su repercusión es marginal en la producción y el consumo de energía y, por ende, impactan poco en la reducción de la vulnerabilidad y de las emisiones de gases de efecto invernadero. Aunque incipientes y parciales, las experiencias se enmarcan en las búsquedas por superar las crisis - tanto la energética como la crisis económica de 2001-y por avanzar hacia un modelo que se reivindica como más limpio, inclusivo y sustentable.

Desde el denominado Consenso de Guayaquil (2002), los estados de la Unasur plantean entre sus once principios rectores, el acceso universal a la energía como un derecho del ciudadano (Unasur y Olade,
2 2011-2013 ANR Sud 2010 Projet Termos, S. Jaglin (responsable) "Trajectoires Energétiques dans les Régions Métropolitaines des Suds” LATTS Laboratoire Techniques, Territoires et Sociétés. tersitarios 30

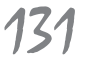


${ }^{3}$ Las situaciones de mayor degradación que primaban en el Conurbano, se agudizan también en la ciudad, donde más del $15 \%$ de los habitantes viven en la calle, en villas de emergencia o están por ser desalojados o más del $10 \%$ de los hogares se encuentran en situación de hacinamiento (Sánchez, 2013).

\section{territarias 30}

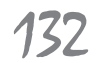

2012). A pesar de que se reconozca la importancia de disponer de energía para la salud y la educación, el avance económico y el bienestar social, en Buenos Aires miles de personas se ven impedidas de acceder a los servicios modernos de energía - electricidad y gas por red-o se deben procurar la energía por vías más costosas y riesgosas.

En toda la Argentina pero fundamentalmente en Buenos Aires, la energía recibe altos subsidios que bajan sensiblemente el costo para los usuarios. Esto eleva significativamente el gasto público, pero en su aplicación se hacen prevalecer criterios socio-políticos a los parámetros de eficiencia energética y económica, porque el problema de la energía no es solo usarla eficientemente sino también disponerla de manera justa y equitativa. A pesar de la aplicación de subsidios y tarifas diferenciadas, la segregación socio-espacial de la población y la precariedad habitacional ${ }^{3}$ tienen su correlato en la inequidad y la pobreza energética, porque muchas personas de bajos ingresos no tienen acceso al gas natural o a la electricidad subsidiados, mientras la mayoría de la población con ingresos medios y altos se beneficia de las tarifas bajas o subsidiadas, despilfarrando a menudo los recursos. Esto se traduce en ausencia de justicia energética, tanto por condiciones desfavorables para muchos, como por el uso abusivo de recursos por parte de otros, también en desmedro de generaciones futuras y del ambiente (Marx, 2011).

\section{Buenos Aires damnificada. Cuando el sistema falla}

El título de esta parte busca parafrasear el de Disrupted Cities. When Infrastructure fails(Graham, 2010) sobre los disfuncionamientos generados por las fallas de infraestructuras y alude a una doble situación de vulnerabilidad estructural. Porque por una parte, a nivel metropolitano, aun cuando se registren tasas de cobertura y de consumo elevados, existe inequidad en el acceso a la energía. Por otra parte, a nivel nacional, la metrópoli ejerce una presión fuerte sobre un sistema de abastecimiento frágil, dependiente de hidrocarburos importados (que agregan el factor geopolítico a los otros aleas), con cortes de electricidad y gas a las industrias por faltantes y cortes no programados por fallas en las infraestructuras, a menudo inadecuadas u obsoletas.

\section{Ineficiencia e inequidad energéticas metropolitanas}

Argentina, y más particularmente la metrópoli de Buenos Aires, se pueden enmarcar dentro de un perfil general de consumo, que no consigue alcanzar las consignas internacionales de "frugalidad energética" (Haëtjens, 2011) lanzadas por organismos como la Agencia Internacional de la Energía, frente a la escasez de hidrocarburos y los altos niveles de contaminación ambiental. Primero, la región metropolitana de Buenos Aires absorbe el $42 \%$ de la energía consumida en el país (Secyt y Gcaba, 2010). Esta concentración se debe no solo a que 
reúne más de un tercio de la población nacional sino también a la aglutinación de gran parte de las actividades industriales de la Argentina. Si bien el crecimiento del consumo ha sido en general exponencial, los distintos sectores han tenido trayectorias diferenciadas. Entre 1986 y 2000, los usos residenciales e industriales se multiplicaron por más de 2 y el uso comercial por 3,5 (Rosenfeld, Discoli y Barbero, 2003).

Históricamente, Argentina ha apoyado su desarrollo y aprovisionamiento energético a través de la expansión extensiva de sus redes de gas y de electricidad que cubren la mayor parte de su superficie. ${ }^{4}$ Así es que el $97,7 \%$ de los hogares metropolitanos tienen acceso a la red eléctrica y el 56,1\% utiliza gas de red para cocinar. En la ciudad de Buenos Aires (con 1,1 millón de hogares), el 99,5\% tienen electricidad por red y el 99,2\% usan gas por red como combustible para cocinar. En el Gran Buenos Aires (con 2,9 millones de hogares), esas tasas llegan a $9 \%$ y $65 \%$, respectivamente. Es decir que en ambos casos superan los niveles nacionales de $9 \%$ y de $51 \%$ (Indec).

Si la concentración de personas y la extensión de los servicios metropolitanos conllevan que la región tenga el mayor consumo residencial del país, este, a su vez, es más alto por los hábitos urbanos y el acceso históricamente barato a la energía porque el consumo energético en la región ha quedado incentivado por la aplicación de subsidios y de precios bajos. Por ejemplo, la tarifa de electricidad para usuarios residenciales de Capital Federal es aproximadamente cuatro veces más baja que la de la Provincia de Buenos Aires, que resulta baja si se la compara con otras regiones del país o del continente. Esto no favorece el uso racional de la energía sino prácticas de consumo energético poco cuidadosas del recurso, lo que se traduce en un despilfarro de combustibles. Además, frente a situaciones de escasez de recursos se aplican cortes en el servicio a las industrias, evitándose la afectación directa de la población y que esta se consustancie con la situación.

Por su parte, las generadoras y las empresas distribuidoras de electricidad y gas en el área metropolitana - bajo jurisdicción del Estado nacional- estiman que los precios y tarifas regulados, deterioran sus resultados operativos. Esta situación incide en el debilitamiento de la inversión y, por ende, de la oferta general en términos de volumen y de calidad de servicio. Porque, por un lado, se hacen menos inversiones en el refuerzo y la extensión de las redes. Por otro lado, tampoco se invierte lo suficiente en el mantenimiento de las redes de distribución de gas y electricidad, que en parte son vetustas, lo que provoca un deterioro en las infraestructuras que incrementa las pérdidas técnicas y las probabilidades de fallas $^{5}$ (Agüero, 2012).

Por ende, por extensión y calidad en el suministro, al interior del espacio metropolitano, aparecen contrastes en el acceso a la energía. En electricidad, las diferencias son mayores en la calidad del servicio. Mientras que en el servicio de gas por red, contrastan más las áreas de los espacios con o sin conexión. Según un estudio realizado por el Banco de Desarrollo de América Latina
4 Incluso el Proyecto de Energias Renovables en Mercados Rurales (Permer) facilita el aprovisionamiento de poblaciones aisladas con energias alternativas, lo que explica que prácticamente no haya uso de leña en el pais.

${ }^{5}$ Los apagones son cortes no programados de suministro eléctrico que se pueden derivar de daños en las lineas y en subestaciones eléctricas, causados accidental o intencionalmente, por fenómenos naturales, desperfectos técnicos o excesos de consumo. Por definición técnica involucra un minimo de 1.000.000 personas/horas. Es decir cuando quedan' 'sin luz' 1000 personas durante 1000 horas (más de 41 dias) o 1000000 de personas durante una hora. Sin embargo, es el impacto en una sociedad lo que determina que un corte sea considerado apagón. En Buenos Aires, por ejemplo, el 15 de enero de 1999 un apagón afectó 500000 personas entre $4 y$ 10 dias; otro, el 23 de octubre de 2004 afectó 600000 personas durante una hora y otro 17 de mayo de 2007 privó de luz a 168000 habitantes por tres horas. Las causas respectivasfueron fallas en una estación transformadora, en un transformador yen una subestación (Agüero, 2012).

territarias 30

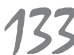


(CAF, 2013), se puede hablar de condición de pobreza energética para referirse al sector de la población que usa el $10 \%$ o más de sus ingresos para pagar sus facturas de energía. Tanto en el acceso a la electricidad como en gas, las situaciones más críticas se encuentran en los barrios más carenciados, que enfrentan una situación de precariedad energética aguda.

En materia de electricidad, por ejemplo, en La Cárcova (José León Suárez, partido de General San Martín), una de las villas más pobres y pobladas del conurbano bonaerense, con 20000 habitantes aproximadamente, solo el $10 \%$ de la población tiene acceso a la red de electricidad por medio de una conexión con medidor propio; mientras que el $90 \%$ restante lo hace a través de la municipalidad que paga sus facturas o recurren a conexiones ilegales (CAF, 2013). Por otra parte, la mayoría de estos habitantes sufren cortes de electricidad recurrentes y como la mayoría - a diferencia de los barrios privados (countries), de las torres-countries o zonas residenciales acomodadas-, no tienen generadores eléctricos propios para cubrir las fallas del sistema. Además, en condiciones precarias de habitación, el consumo de energía resulta además muy poco eficiente por mayores pérdidas (ambientes oscuros, húmedos, cerramientos pobres) y peores prácticas de consumo como calentamiento eléctrico del agua por falta de gas natural. La imposibilidad de acceder al gas natural redunda en un costo mayor, especialmente para la gente de bajos recursos, que no pueden comprar territarias 30 envases grandes y que además tienen que enfrentar otras dificultades para acceder a las garrafas, además de las económicas, como dificultades para obtener botellas de buena calidad para transportarlas.

Pero, en general, en Argentina, buena parte de la energía se pierde también o se desaprovecha en los edificios - especialmente en su acondicionamiento climático- porque pocas veces está contemplada en el diseño. La orientación de los ambientes, las aislaciones térmicas, los materiales, la apertura de vanos, la ubicación de los artefactos y las instalaciones suelen no ser los apropiados para hacer un uso eficiente de la energía (Baragatti, 2010; Edenor, Maggio, 2010; Fovisee, 2012).

Con recursos cada vez más escasos y costosos y con un consumo cada vez mayor, menos cuidadoso y eficiente, Buenos Aires se encuentra frente al desafío de transformar los sistemas de producción y abastecimiento energético.

\section{Un país a merced de recursos escasos}

Más allá de la red de distribución de combustibles líquidos, repartida entre la empresa semiestatal YPF y otras empresas privadas, entre ellas algunas transnacionales presentes en el país desde principios del siglo $\mathrm{XX}$, la red de gas y la red de electricidad son los dos sistemas de aprovisionamiento energéticos principales de la metrópoli. Si bien están fuertemente imbricados física, económica y políticamente, los sistemas tienen evoluciones y rasgos diferenciados.

La energía eléctrica en la ciudad de Buenos Aires, se produce en las centrales Puerto, Puerto Nuevo y Costanera. Justo 
sobre el límite con la ciudad, pero del lado de provincia, se encuentra la central de Dock Sud. Como esquematiza el mapa l, una línea de $500 \mathrm{kV}$ forma un anillo periférico que conecta las centrales de La Plata, el Dique, CMs y Ensenada Fuerte

Mapa 1. Las redes de energía en la metrópoli bonaerense

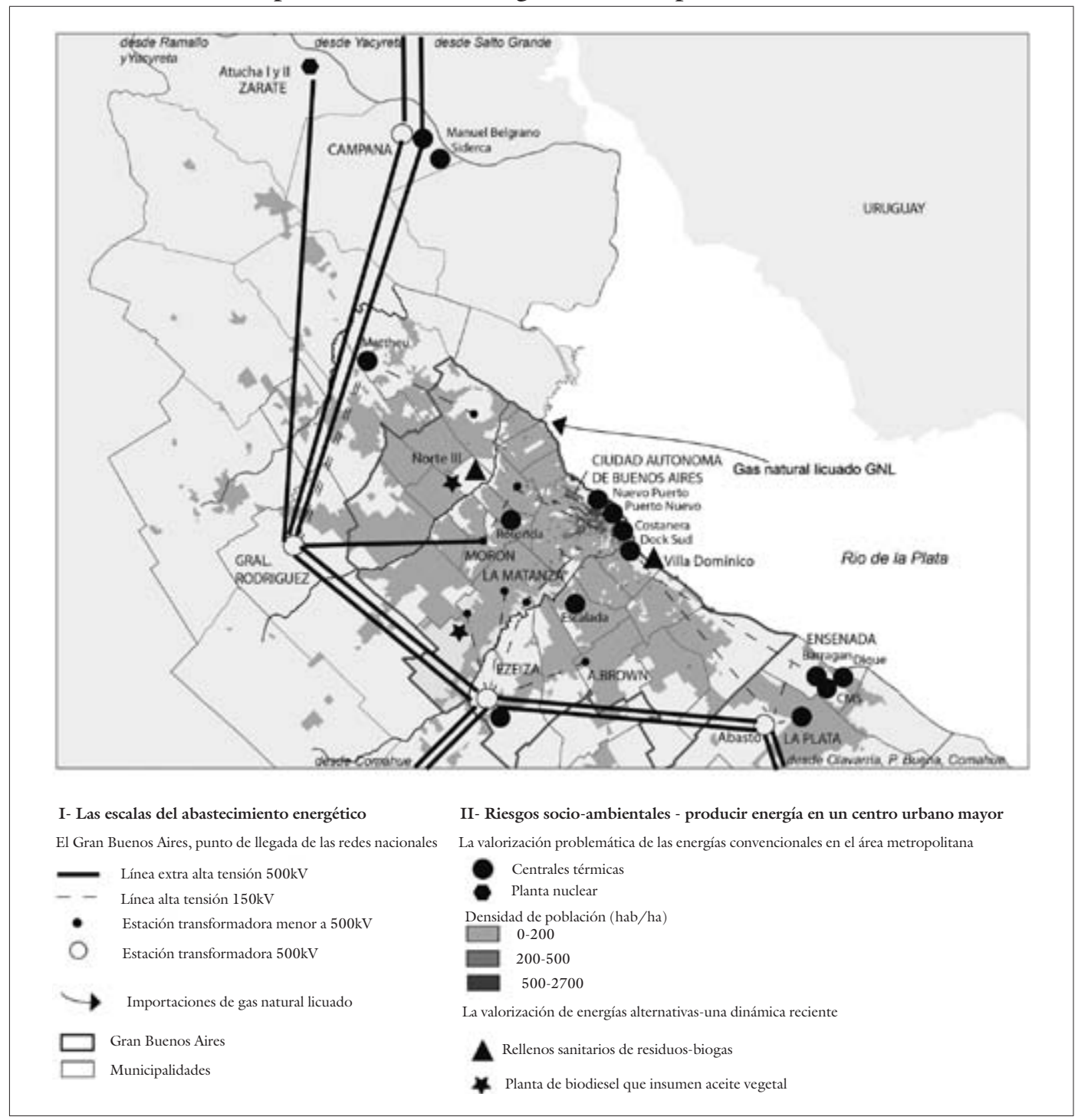

Fuente: Elaboración propia.

Vulnerabilidad energética en la Metrópoli de Buenos Aires territarias 30 
${ }^{6}$ El petróleo aporta $32 \%$, la hidroelectricidad $12 \%$, el carbón y el combustible nuclear $2 \%$ cada uno y otras energias renovables consiguen sumar $1 \%$.

${ }^{7}$ En el año 2011, se pagó alrededor de US\$2 MBTU y entre US $\$ 10$ y US $\$ 16 / M B T U$ de gas importado desde Bolivia o gas natural licuado GNL traido desde paises distantes.

\section{territarias 30}

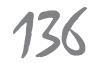

Barragán al sur de la región metropolitana con las centrales Geneba al oeste, en Ezeiza y Matheu al norte, próxima a General Rodríguez. A este primer cordón quedan conectadas las centrales del corredor industrial fluvial, en Ramallo-San Nicolás, Zarate y Campana. En este partido se encuentra en funcionamiento la central nuclear Atucha I y debería entrar en operación, en 2014, Atucha II (Carrizo y Forget, 2011). La generación eléctrica dentro de la metrópoli es térmica, utilizándose gas natural, gas oil y fuel oil. El uso de este último combustible ha crecido en los últimos años, lo que provoca un aumento de las emisiones de gases de efecto invernadero. Pero, aun cuando la electricidad producida en la metrópoli es considerable, la región importa electricidad a través de las líneas de alta tensión del sistema interconectado nacional, particularmente de las grandes centrales hidráulicas del Comahue o el litoral fluvial y por las conexiones internacionales puede comprar electricidad a países vecinos.

El gas que la metrópoli consume lo importa en su totalidad, por gasoductos desde las cuencas argentinas y desde Bolivia o por vía marítima, de países distantes. Argentina depende energéticamente del gas que cubre un $51 \%$ de sus necesidades energéticas ${ }^{6}$ (BP, 2010). El uso creciente y diversificado del gas en el país se inició décadas atrás, promovido por la empresa Gas del Estado que extendía las redes de transporte y distribución; se reforzó mediante un programa del Ministerio de Planificación que incentivó la conversión de autos y se acentuó en la década de 1990, por las inversiones priva- das en generación eléctrica que buscaban eficiencia para ganar en el mercado. Hacia mediados de los años 1990, el gas superó al petróleo como fuente de energía primaria en Argentina. Luego se convirtió en la mayor fuente de electricidad. En 2005, el $47 \%$ de la generación fue producida a gas, cuando la hidroelectricidad cubrió el $36 \%$ de las necesidades. En 2011, un tercio del gas entregado en el país (110 millones de $\mathrm{m}^{3} /$ día) fue destinado a las centrales térmicas, otro a la industria y otro se repartió entre usuarios residenciales (24\%) y el parque automotor a gas natural comprimido (7\%) (Enargas, 2011).

A pesar de la dependencia histórica y creciente que el país tiene de los hidrocarburos, la explotación de los recursos nacionales no se ha hecho de manera sostenible. En las dos últimas décadas, las reservas de gas y de petróleo han descendido abruptamente. Los precios que se pagan por los hidrocarburos producidos en Argentina son muy inferiores a los que se pagan por los hidrocarburos importados, ${ }^{7}$ lo que vuelve menos atractiva la actividad en el país, particularmente las inversiones a riesgo necesarias para ampliar las reservas. Entre 2004 y 2011, las principales empresas que operan en el país - a excepción de Pan American Energy - vieron bajar su producción y reservas probadas de petróleo. En gas, producción y reservas también fueron disminuyendo para la mayoría de las empresas: la china Sinopec y la francesa Total fueron las únicas que pudieron aumentar considerablemente su producción. La del principal yacimiento gasífero del país Loma 
La Lata-Sierra Barrosa declina desde el año 2004 (Secretaría de Energía, Fundación Bariloche y Reeep, 2009).

El incremento de la demanda energética exigió un aprovisionamiento mayor en combustibles fósiles. Pero la explotación de las cuencas hidrocarburíferas argentinas - ya maduras - no logró cubrir las necesidades nacionales. El país volvió a perder el autoabastecimiento y debió recurrir a las importaciones de gas (figura 1), ya no solo de Bolivia, sino también de cuencas distantes comprando gas natural licuado GNL. Las importaciones de Bolivia han ido creciendo. Superan los tres mil millones de $\mathrm{m}^{3}$ en 2011 (9 millones $\mathrm{m}^{3} / \mathrm{d}$, aproximadamente) pero están limitadas por la capacidad del gasoducto existente, hasta tanto se concrete el proyecto del gasoducto de noreste, recurrentemente postergado. Las importaciones por vía marítima también fueron aumentando. El gas natural licuado entra desde el año 2008 por el puerto del complejo MEGA en Bahía Blanca (12 millones $\mathrm{m}^{3} / \mathrm{d}$ en promedio) y desde el año 2011, también por Escobar (14 millones $\left.\mathrm{m}^{3} / \mathrm{d}\right){ }^{8}$ Existiría a futuro la posibilidad de desarrollar las cuencas inexploradas y de explotar los recursos de gas no convencional, que posicionan a Argentina entre los tres más ricos en materia de recursos, junto a China y Estados Unidos ${ }^{9}$ (774 trillones de pies cúbicos de recurso recuperable), con gran potencial en la cuenca de Neuquén pero también en la Austral-Magallanes y en el golfo de San Jorge (us Energy Information Administration, 2011).

El gas que la metrópoli consume lo importa en su totalidad, por gasoductos desde las cuencas argentinas y desde Bolivia

Figura 1. Exportaciones e importaciones de combustibles (millones de dólares corrientes)

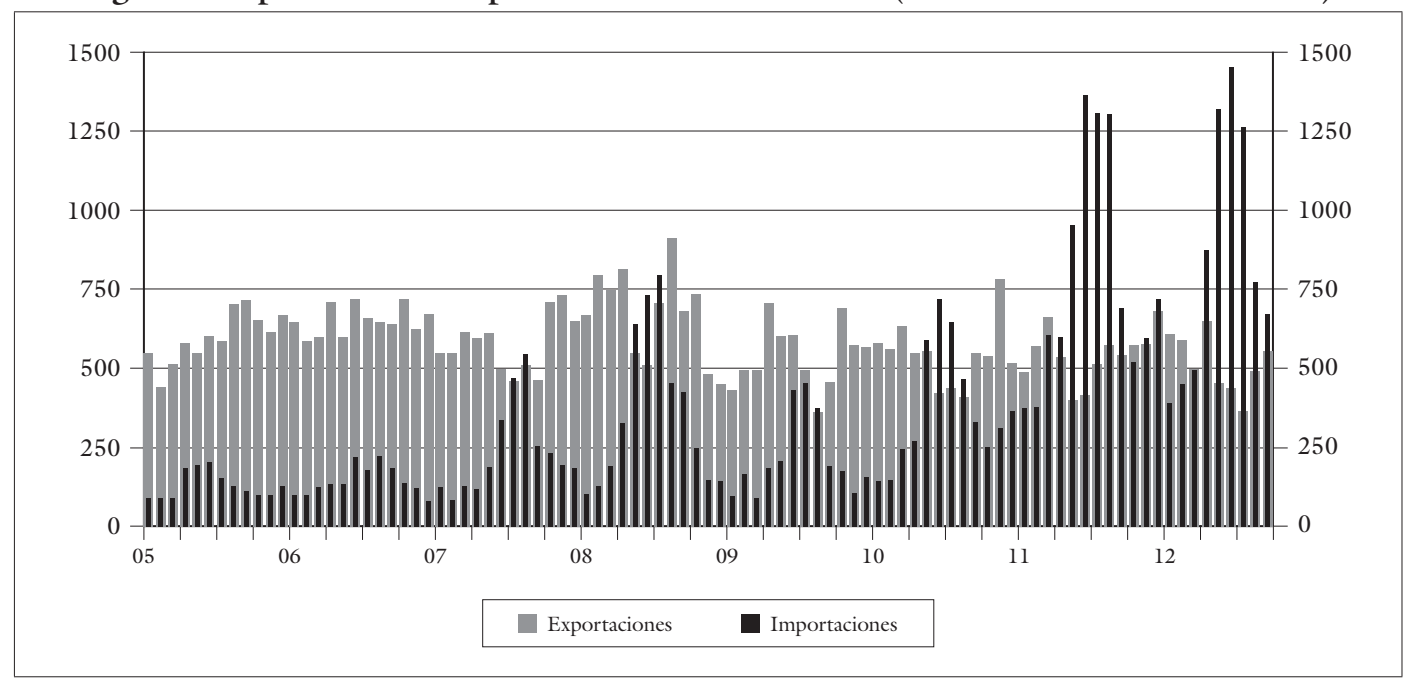

Fuente: Elaboración propia a partir de datos de Indec. 
${ }^{10}$ Las provincias productoras de soja (no productoras de petróleo) manifestaron disconformidad frente a su exclusión de su participación en YPF. Parte del reclamo se basa en que el Fondo Solidario de la Soja (que recupera $30 \%$ de las retenciones, equivalente a $\$ 7000$ millones en 2011) es distribuido entre el conjunto de las provincias (en base a los coeficientes establecidos por ley de coparticipación).

\section{territarias 30} 138 o por vía marítima, de países distantes. En 2013 , como en años anteriores, se ha recortado en ciertas oportunidades el servicio interrumpible, es decir, aquel por el que las compañías pagan una tarifa menor, aceptando exponerse a cortes cuando escasea el fluido. Pero en casos de emergencia, que ocurren periódicamente durante el invierno y el verano, cuando hay altos picos de demanda, también quedó afectado el aprovisionamiento de algunas otras industrias, fundamentalmente en la ciudad de Buenos Aires y las provincias de Buenos Aires, La Pampa y Santa Fe.

Es en este contexto de escasez energética creciente - con faltantes de gas, de combustibles líquidos y con riesgos mayores de blackout-, que en el año 2012, algunas provincias revocaron concesiones de áreas petroleras y el gobierno de la presidenta Cristina Fernández, expropió la empresa YPF, que la española Repsol había adquirido durante el gobierno del presidente Carlos Ménem en la década de 1990. $51 \%$ de las acciones de YPF sujetas a expropiación pasan a manos del Estado nacional y el $49 \%$ restante, a las provincias integrantes de la Organización Federal de Estados Productores de Hidrocarburos. ${ }^{10}$

\section{Resistencia energética. ¿Resiliencia metropolitana?}

Frente a los problemas energéticos de inequidad e inseguridad en Buenos Aires, y en el marco de los debates internacionales que promueven la resiliencia urbana y la adaptación al cambio climático, surgen propuestas y proyectos para una producción más limpia y sustentable, y un uso más eficiente de la energía, introducidas o promovidas desde las escalas nacional, provincial o local. Fuera de las pequeñas iniciativas individuales de instalación de paneles fotovoltaicos, colectores solares o de producción de bioenergía - de las que no se dispone información sistematizada-, las principales estrategias promovidas institucionalmente con impacto en la metrópoli procuran fundamentalmente: 1) aumentar la oferta energética a partir del aprovechamiento de los residuos metropolitanos y 2 ) eficientizar el uso de los recursos.

\section{Los residuos para una energía "limpia"}

En el área metropolitana de Buenos Aires, en los años setenta, para solucionar la proliferación de basurales a cielo abierto y la incineración mal controlada de residuos, se proponía utilizar los residuos como material de relleno de zonas anegadizas. A partir de los años noventa, la preocupación ambiental que se difunde con la Cumbre de la Tierra en Río de Janeiro (1992) y luego con el Protocolo de Kyoto (1997), conlleva a revisar la disposición de residuos, considerada responsable por la emisión de un $30 \%$ de gases de efecto invernadero.

En 2002, se abre una sección 'Residuos' en la Oficina Argentina para Mecanismos de Desarrollo Limpio (MDL) conformada en 1999, para desarrollar métodos de captación del metano emitido por los rellenos sanitarios, y cuyo potencial de efecto invernadero es considerado 21 veces más 
alto que el dióxido de carbono (Blanco, 2004). Varias empresas extranjeras, entre ellas las holandesas (Villa Domínico-Van der Wildt) e italianas (Norte III) desarrollaron redes de extracción, monitoreadas por una comisión de Naciones Unidas. La captación del gas metano en los rellenos sanitarios de Villa Domínico y Norte III marca una inflexión en las modalidades de revalorización de los residuos que se venía promocionando (fotos 1 y 2 ). Sin embargo, estas innovaciones tuvieron un impacto limitado. En Villa Domínico, la implementación tardía de estos dispositivos hace que las cantidades de metano captadas sean muy

Foto 1. Planta de extracción y combustión de metano del relleno sanitario de Norte III

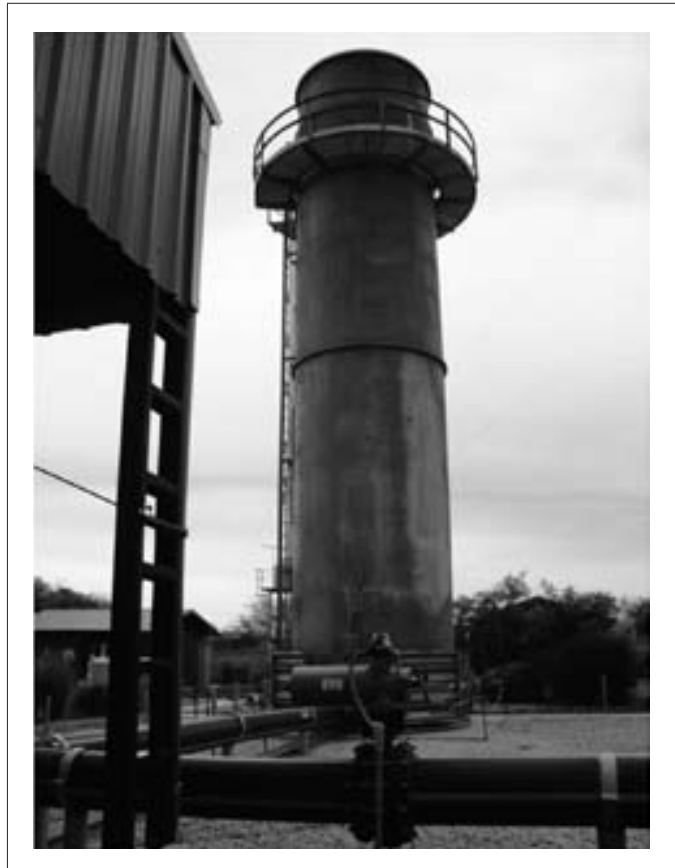

Fuente: Marie Noelle Carré, 2012. pocas, la gran parte de este gas ya se diluyó en la atmósfera. En Norte III, la turbina generadora de electricidad se activó en 2012 y todavía es demasiado temprano para evaluar su eficiencia. La previsión de generación de bonos de carbono es, en cambio, bastante ambiciosa: un millón y medio en Norte III, en diez años. Si bien todavía no probó completamente su eficiencia, este procedimiento se difundió como 'buena práctica' en otros puntos de la Argentina.

En 2006, en la Ciudad Autónoma de Buenos Aires, fue promulgada la Ley 1854 de Gestión Integral de Residuos Sólidos Urbanos, llamada 'Basura Cero' y ese mis-

Foto 2. Procesamiento de aceite vegetal usado en la planta de RBA Ambiental en San Miguel

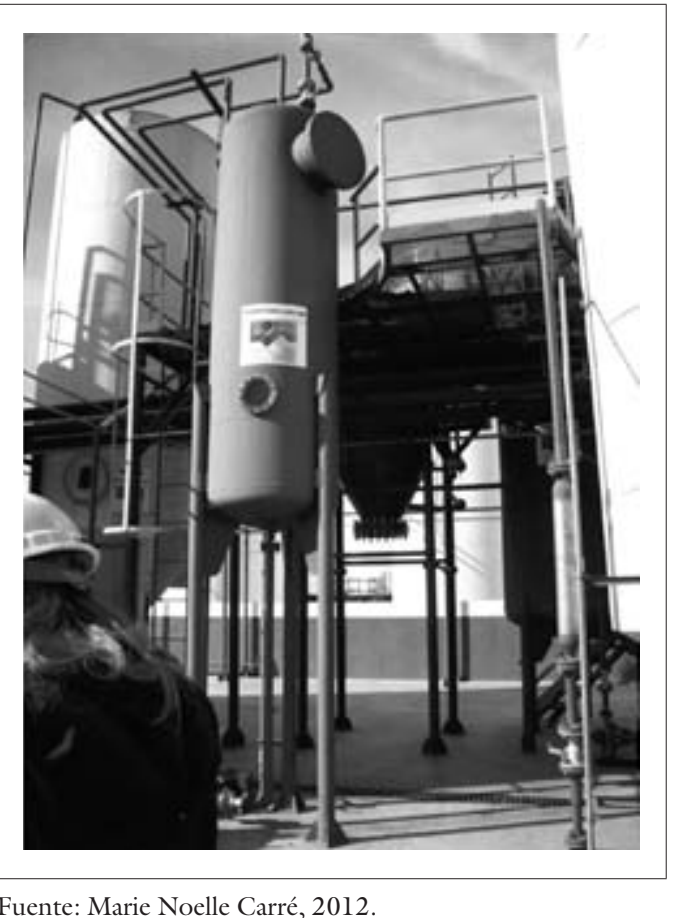

territarias 30

Vulnerabilidad energética en la metrópoli de Buenos Aires 
${ }^{11}$ Los cartoneros son recuperadores urbanos que seleccionan los materiales reciclables en los residuos de Buenos Aires y los revenden para sobrevivir. En esta urbe, la aparición masiva de recuperadores es concomitante con la crisis de 2001. Desde aquella fecha, una parte de estos actores logró organizarse en cooperativas y formalizar progresivamente su actividad.

\section{territarias 30} 140 mo año, la provincia de Buenos Aires promulgó la Ley 13592 de Gestión Integral de Residuos Sólidos Urbanos (GIRSU). Esta legislación apunta a responsabilizar a cada territorio político-administrativo por la gestión de sus residuos, alentando a que esta sea más participativa, sustentable y comunitaria. Pero hasta ahora, prácticamente no hay políticas públicas de separación y de gestión diferenciada de residuos, excepto la actividad informal que vienen llevando a cabo los recuperadores urbanos. ${ }^{11}$

Entre los casos excepcionales, el programa de recuperación de Aceite Vegetal Usado (AVU) de la provincia de Buenos Aires valorizaría los residuos para la producción de biocombustibles. El biodiesel producido sería destinado al mercado de combustibles líquidos, ya para transporte, ya para generación eléctrica. La empresa Ecopor, por convenio con la ciudad de Buenos Aires y los municipios de la provincia de Buenos Aires, recuperaría el aceite vegetal usado de los locales de restauración o en puntos de aporte voluntario y acopio comunitario, en la mitad de los locales de ciudad de Buenos Aires y en 92 municipios de la provincia, centralizando y redistribuyéndolo (30 toneladas por día) - previa filtración- entre empresas de producción de biodiesel y se entregaría el producto a centros comunitarios de los municipios que participan.

Otro ejemplo lo constituye el proyecto del Centro Ambiental de Recomposición Energética (CARE), desarrollado por la municipalidad de la Matanza, en convenio con Energía Argentina Sociedad Anónima
(Enarsa). Se ubica hacia el Sur del municipio - el más poblado y pobre del conurbano bonaerense-, un sitio estratégico para el marketing metropolitano. El Estado nacional financia, en este espacio denso del peronismo en la Provincia, un equipamiento considerado una innovación 'única en América Latina' (ver video en http:/ / www. youtube.com/watch?v=mxX_YJYZSq4). El municipio ve una oportunidad de promover su dinamismo en términos de creación de empleo, de independizar al municipio de las infraestructuras metropolitanas de gestión de residuos y que lo integraría dentro del mercado de los bonos de carbono. Este emprendimiento reposa sobre el método de la depolimerización catalítica, un proceso de cracking de las cadenas de carbono utilizado en la industria petrolera. Este método todavía no ha sido puesto a prueba y permanece en un estado experimental a nivel mundial: según los estudios existentes, es valioso para la transformación de cauchos, pero en el caso de la Matanza se propone usarlo para el aprovechamiento de residuos domiciliarios compuestos por una gran diversidad de sustancias. Las dudas sobre la naturaleza del procedimiento técnico empleado, llevó a la sospecha de que sería un centro de incineración con revalorización energética (método de tratamiento de los residuos prohibido en 1977). Por lo tanto, un grupo de habitantes, aunque bastante dificultado por su aislamiento físico y socio-económico - a más de 40 kilómetros del centro de la ciudad, en un margen pobre de la Matanza- logró captar una cobertura mediática con el apoyo de 
grupos más grandes. La implementación del CARE desencadenó un conflicto que, a primera vista, podría ser calificado de conflicto de cercanía, pero que, de hecho, se ubica dentro de un cuestionamiento más amplio sobre las modalidades del desarrollo sustentable.

El reciclado de residuos y su aprovechamiento para la generación eléctrica o producción de combustibles constituirían una solución ambiental que reduzca el daño causado por los déficits en el tratamiento de residuos domiciliarios en la mayor aglomeración urbana del país y una alternativa energética. Por ende, como una doble respuesta a la crisis del sistema sociotécnico de la gestión de los residuos y a la de los sistemas de abastecimiento energéti$\mathrm{co}$, resultan un buen punto de partida para pensar la resiliencia.

\section{En pos de un uso eficiente de la energía}

A la problemática de incorporación de equipamientos promocionados en pos de un desarrollo sustentable, se superpone una estrategia nacional de uso racional y eficiente de la energía. Este fue declarado de interés y prioridad nacional, considerándoselo un componente imprescindible de la política energética y de la preservación del medio ambiente (Argentina, 21 diciembre 2007).

Hacer uso eficiente de la energía no significa consumir menos sino consumir mejor, manteniendo las mismas prestaciones y utilizando la tecnología adecuada. Por ejemplo, se estima que el gas consumido anualmente por los usuarios residenciales se emplea en un $60 \%$ para calefacción y en un $40 \%$ para calentamiento de agua y cocción (estos porcentajes varían según la temperatura que haga en el año). Pero casi todos los usuarios de gas - no solo residenciales, sino también comerciales y otros- disponen de al menos un piloto encendido que consume unos $0,5 \mathrm{~m}^{3} /$ día (equivalentes a 225 Watts) y la mayor parte de tiempo ese gas se quema sin ser aprovechado; en total representaría más de 3,5 millones de $\mathrm{m}^{3}$ de gas por día, es decir, una cifra que se aproxima a las importaciones diarias de gas boliviano (2009). Si los pilotos fueran reemplazados por sistemas de encendido electrónico, el ahorro de gas tendría amplios beneficios energéticos, económicos y ambientales (Gil, 2011). Con el menor consumo de gas, se reducirían los faltantes, las importaciones y las emisiones, y, por ende, la vulnerabilidad.

En concordancia con la idea de que se consuma energía más eficientemente, se han tomado medidas que impulsan el mejoramiento de los artefactos y de las construcciones o tienden a ello:

- Se exige el etiquetado de algunos artefactos eléctricos, obligando, de ese modo, a fabricar y comercializar productos de mayor calidad y a usar la energía de manera más eficiente. En 1998, el Instituto Argentino de Normalización y Certificación (IRAM) publicó la primera norma de eficiencia energética: IRAM 2404-3 para heladeras. La misma tuvo aplicación a partir del año 2005 , con la territarias 30 141 
12 Enargas sería quien se ocupe de lo concerniente a los artefactos de cocción a gas, calefones y termotanques.

territarias 30 142
Resolución 35 de Lealtad comercial de la Secretaría de Coordinación Técnica General que impuso la certificación del producto y la exhibición de la etiqueta de eficiencia energética en cada artefacto comercializado. En el año 2009, la Secretaría de Energía establece niveles máximos de consumo específico de energía o mínimos de eficiencia energética para la comercialización de refrigeradores de uso doméstico (Resolución 396). La Secretaría de Energía consigue que se estudie, norme y aplique -en vinculación con dichas instituciones - el etiquetado obligatorio de lámparas incandescentes y fluorescentes; de acondicionadores de aire y de lavarropas. Además el Instituto Argentino de Normalización y Certificación estableció las normas para motores eléctricos de inducción trifásicos y los balastos de lámparas fluorescentes. ${ }^{12}$ Están en estudio las normas para los stand by, las electrobombas para elevación de agua, termotanques eléctricos y televisores. En cada caso se siguieron las clasificaciones de la Unión Europea. La implementación de estas medidas requiere la existencia en el país de laboratorios equipados y acreditados, capaces de acompañar el desarrollo. Pero la puesta en marcha ha tenido respuesta rápida de parte de los consumidores.

- Se trabaja en mejorar el diseño de la construcción y la calidad de los materiales desde distintos ámbitos (INTI, universidades, ONG). Se busca lograr un uso sustentable de la energía en los edificios desde la fabricación y disposición de los materiales hasta las prácticas en el habitar. Si bien el etiquetado de viviendas no está implementado, se ha comenzado a etiquetar materiales o partes, para mejorar la eficiencia energética en un edificio; por ejemplo IRAM avanza en el etiquetado de ventanas (IRAM 11507-4). En la Provincia de Buenos Aires, la Ley 13059/2003 establece el cumplimiento obligatorio de nueve normas IRAM de eficiencia energética de envolventes en la construcción, aunque tal cumplimiento haya sido escaso (Kozak, Romanello, 2012).

- Desde la Nación, se encaró el Programa de ahorro y eficiencia energética para edificios públicos (PAyEeEP Ley 3246 de 2009 y Decreto 300 de 2010) que busca optimizar el consumo de 1500 edificios públicos de la ciudad, reduciéndolo a un $30 \%$. Con este objetivo se nombraría un administrador energético en cada edificio.

- A escala de Buenos Aires, el gobierno de la ciudad publicó un modelo territorial 2010-2060 que se basa en un plan urbano ambiental (Ley 2930 de 2008) y la ley de adaptación y mitigación del cambio climático (3871 de 2011). Contempla el desarrollo de la 'ciudad sustentable', es decir aquélla que integra elementos para mejorar el impacto sobre el medio ambiente, aumentar la eficiencia energética y la resiliencia urbana. En 2012, la ciudad aprobó la Ley 4024 de sistemas de captación de 
energía solar - aun sin reglamentación- que establece un régimen de incentivos para su uso y a través de la APra Agencia de Protección Ambiental de la Ciudad ha desarrollado varias operaciones con el objetivo de medir la calidad ambiental, ${ }^{13}$ promover incentivos para actores con metas ambientales ${ }^{14}$ y reducir la precariedad energética de los habitantes de bajos ingresos.

Este panorama ilustra que en términos de energías alternativas y de uso racional, existen discursos e ideas para el cambio; $\sin$ embargo, las medidas implementadas y los emprendimientos concretados son bastante puntuales y sus impactos son residuales. Porque no siempre el marco normativo se ha traducido en la concreción de las políticas, ni en el avance hacia las metas. Se han dado fundamentalmente iniciativas individuales, parciales y desarticuladas, sin un plan integral promovido a una escala colectiva y sin poder generar una transición hacia otro modelo más limpio y sostenible, mientras el aumento del consumo de energía sigue creciendo rápidamente y la vulnerabilidad con él.

\section{Reflexiones finales}

En Argentina, la política de abastecimiento energético basada en el uso de gas y derivados de petróleo, mostró sus fallas superlativas, cuando el país tuvo que reiniciar las importaciones de hidrocarburos para poder sostener el consumo creciente - mayor por usuario y en número de usuario- en un marco de crecimiento económico nacional. Pero las políticas públicas no tienden a modificar el modelo energético actual sino a reforzarlo y agravan la vulnerabilidad energética por la exposición al alea y por la consolidación de un espacio carente de alternativas (Dauphiné y Provitolo, 2007).

El black out del 7 noviembre de 2012 que afectó más de tres millones de habitantes de la metrópoli de Buenos Aires y la gran inundación, días después (La Nación, 2012), confirmaron el estado de precariedad de las infraestructuras y revelaron la necesidad de aumentar la resiliencia metropolitana y de preparar la población y el territorio para casos de emergencia.

Si bien hubo algunos avances en materia de uso racional y eficiente de la energía, los intentos implementados dentro de la ciudad autónoma de Buenos Aires, encuentran pocas posibilidades de réplica en el conurbano, donde la impulsión económica y el acompañamiento técnico para el cambio padecen las limitaciones presupuestarias de los municipios. En el conurbano, en cambio, aparecen espacios con posibilidades de innovación. Sin embargo, mayormente Buenos Aires se sigue desarrollando con un sistema de infraestructuras centralizadas que trabajan al límite y poblaciones que no se preparan para consumir mejor, ni para enfrentar las crisis, que se vuelven cada vez más posibles. No se acondiciona el sistema en función de la vulnerabilidad que existe; vulnerabilidad que aumenta, en buena medida por el crecimiento acelerado
${ }^{13}$ En 2010, se inauguró, en Villa Soldati, el Centro de Información y Formación Ambiental (CIFA) que tiene por misión estudiar la calidad del aire y del agua y buscar soluciones concretas para enfrentar los desafios ambientales.

${ }^{14}$ El programa 'Buenos Aires produce más limpio' recompensa con financiamiento, a emprendedores con conciencia ambiental.

territarias 30

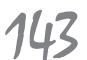


del consumo que es fomentado por la estructura tarifaria y un acceso amplificado a productos energívoros.

Aunque se ha trabajado de forma desarticulada y puntual, existen dificultades y barreras para implementar cambios (Secretaría de Energía, Fundación Bariloche y Reeep, 2009) y no se ha encarado el tema de la pobreza e inequidad energética, el desafío energético exige pensar en reducir la vulnerabilidad metropolitana y desarrollar resiliencia. Se trata de un desafío múltiple: 1) poder atender el aumento de demanda de energía que acompaña el crecimiento económico; 2) poder dar respuesta a las posibles interrupciones o fallas de grandes sistemas técnicos, que superan ampliamente las escalas de las urbes (Dupuy y Tarr, 1988); 3 ) limitar esa posibilidad de devenir una "ciudad damnificada u obstaculizada" (Graham, 2010), previniendo un colapso macro-regional y acotando la dependencia externa y 4) restringir el uso de los combustibles fósiles y promover el uso de fuentes de energías alternativas. Estas acciones servirían para aumentar la capacidad de los sistemas y la disponibilidad de recursos, reduciendo la vulnerabilidad metropolitana. El desafío es también una oportunidad para abrir el debate, intervenir en pos de la justicia energética y adecuar las políticas implementadas con los discursos que se lanzan desde distintas instancias, sobre las precauciones y estrategias a desarrollar frente al cambio climático.

\section{Referencias}

Agüero, J. (2012). Apagones: ¿Es posible evitarlos? Disertación en el acto de su incorporación como Académico Titular de la Academia de la Ingeniería de la Provincia de Buenos Aires. Recuperado de http://acaingpba.org.ar/Conferencia \%20Incorporacion $\% 20$ Jorge $\% 20$ Aguero\%2017-5-2012.pdf

Argentina, Ministerio de Planificación Federal, Inversión Pública y Servicios. Decreto 140 (21 diciembre 2007).

Baragatti, A. (octubre, 2010). Eficiencia energética. El programa argentino: avancesy expectativas. Ponencia presentada en el III Seminario para la creación de un Foro Permanente de Eficiencia Energética en América Latina. Ciudad de México.

Blanco, G. (coord.). (2004). Contratos de reducciones de emisiones (Ers) provenientes de proyectos de manejo de residuos sólidos urbanos Reporte de la asistencia técnica. Buenos Aires: Ceamse.

Blaikie, R., Cannon, T., Davis, I. y Wisner B. (1996). Vulnerabilidad. El entorno social, político y económico de los desastres. Bogotá: Tercer Mundo Editores.

CAF. (2013). Energía: una visión sobre los retos y oportunidades en América latina y el Caribe. Aspectos sociales del acceso a la energía. Documento de trabajoborrador para discusión y análisis. Recuperado de http://www.caf.com/_custom/static/agenda_energia/assets/ caf_agenda_energiat6_sociales.pdf

Carrizo, S. y Forget, M. (jan/jun, 2011). Aprovisionamiento eléctrico de Buenos 
Aires y desigualdades regionales entre la metrópolis y el Noreste argentino. Sustentabilidade em Debate, 2,(1), 33-50.

Dauphiné, A. y Provitolo, D. (2007). La résilience-un concept pour la gestion des risques? Annales de Géographie, (654), 115-125.

D’Ercole, R. y Metzger, P. (2009). La vulnérabilité territoriale: une nouvelle approche des risques en milieu urbain. $C y$ bergeo, (447). Recuperado de http:// cybergeo.revues.org/22022

Di Meo, G. (1998). Géographie sociale et territories. Paris: Nathan.

Dupuy, G. y Tarr, J. A. (éd.) (1988). Technology and the rise of the networked city in Europe and America. Philadelphia: Temple University Press.

Edenor, D. y Maggio, N. (2010). Proyecto: Casas por + energía. Revista CIER, (54), 42-49.

Enargas (2011). Informe anual Enargas 2011. Recuperado de www.enargas. gov.ar

Fovisee. (2012). Manual de instrucciones para el uso sustentable de la vivienda. Buenos Aires: Autor.

Gil, S. (2011). Una mirada al ahorro energético. Serie hojitas de conocimiento Instituto de energía y desarrollo sustentable. Buenos Aires: Comisión Nacional de Energía Atómica. Recuperado de file:///C:/Users/toshiba/Downloads/53-54_HojitaCNEA_N2011_ GIL_Pilotos_FINAL-libre\%20(1).pdf

Graham, S. (ed.) (2010). Disrupted cities. When Infrastructure Fails. London: Routledge.
Haëtjens, J. (2011). La ville frugale. Un modèle pour préparer l'après-pétrole. Limoges: FYP éditions.

Hertzer, H. (1990). Los desastres no son tan naturales como parecen. Medio Ambiente y Urbanización, 8(30), 10-30.

Instituto Nacional de Estadísticas y Censos. (2010). Censo Nacional de Población, Hogares y Viviendas. Recuperado de http://www.censo2010.indec.gov. ar/cuadrosDefinitivos/analisis_censo_febl2.pdf

Kozak, D. y Romanello, L. (2012). Sustentabilidad II: Criterios y normativas para la promoción de sustentabilidad urbana en la CABA. Buenos Aires: CPAU.

La Nación (2012, 7 de noviembre). El apagón en Buenos Aires afectó a tres millones de personas y muchos barrios estuvieron sin luz. La Nación. Recuperado de http://www.lanacion.com. ar/1524381-el-apagon-en-buenos-aires-afecto-a-tres-millones-de-personasy-muchos-barrios-estuvieron-sin-luz

Marx R. (septiembre, 2011). Energía: una cuestión de justicia. Diálogo politico, XXVIII(3). Recuperado de http://kas. org.ar/DialogoPolitico/pdfs/09_dossier.pdf

Rosenfeld, E., Discoli, C. y Barbero, D. (2003). El consumo de energía en el area metropolitana de Buenos Aires en la década del 90: una trayectoria de desarrollo insustentable. Avances en Energias Renovables y Medio Ambiente 7(1). Recuperado de http:// asades.org.ar/modulos/averma/ trabajos/2003/2003-t007-a001.pdf territarias 30

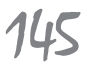


Sánchez, S. (2013). Acciones de resiliencia frente a las políticas habitacionales en la ciudad de Buenos Aires: las organizaciones sociales, las instituciones y los desalojos masivos. Territorios (28), 79-104.

Secretaría de Energía, Fundación Bariloche y Reeep. (2009). Energias Renovables. Diagnóstico, barreras y propuestas. Buenos Aires: Autor.

Unasur y Olade (2012) Unasur: un espacio que consolida la integración energética. Recuperado de http://www.iadb.org/ intal/intalcdi/PE/2012/10228.pdf

us Energy Information Administration. (2011). World shale gas resources: an initial assessment of 14 regions outside the United States. Washington: us Department of Energy.

\section{Webgrafía}

Secyt y Gcaba. (2010). Atlas Ambiental de Buenos Aires http://www.atlasdebuenosaires.edu.ar

BP https://www.bp.com/
C40 Cities Climate Leadership Group http://live.c40cities.org/about

CARE Centro Ambiental de Reconversión Energética La Matanza http://www. youtube.com/watch?v=mxX_YJYZSq4

Clinton Climate Initiative www.clintonfoundation.org

INDEC http://www.indec.mecon.ar/

Local Governments for Sustainability www. iclei.org

Pacto de la ciudad de México http://www. mexicocitypact.org/en/the-mexicocity-pact-2/

Secretaría de Energía. Argentina http:// energia3.mecon.gov.ar

The Carbon Disclosure Project www.cdproject.net

us Energy Information Administration http://www.eia.gov

UNISDR Desarrollando ciudades resilientes http://www.eird.org/camp-10-15/ index.html 